\title{
An integrated approach to improving the availability and utilisation of tuberculosis healthcare in rural South Africa
}

\author{
N Chimbindi, T Bärnighausen, M-L Newell \\ Africa Centre for Health and Population Studies, University of KwaZulu-Natal, Durban, and School of Public Health, Faculty of Health Sciences, \\ University of the Witwatersrand, Johannesburg, South Africa
}

N Chimbindi, MSc

Africa Centre for Health and Population Studies, University of KwaZulu-Natal, Durban, and Department of Global Health and Population, Harvard School of Public Health, Boston, MA, USA

T Bärnighausen, $\mathrm{MD}, \mathrm{ScD}$

Africa Centre for Health and Population Studies, University of KwaZulu-Natal, Durban, and Centre for Paediatric Epidemiology and Biostatistics, Institute of Child Health, University College London, UK

M-L Newell, $\mathrm{PhD}$

Corresponding author: N Chimbindi (nchimbindi@africacentre.ac.za)

\begin{abstract}
Background. Patients with tuberculosis (TB) face several challenges in accessing care, and an integrated service that includes HIV testing could be preferable for them and ensure timely HIV treatment initiation and optimal TB care.

Objectives. To investigate factors, including uptake of HIV testing, associated with availability and utilisation of healthcare by TB patients in a rural programme devolved to primary care in Hlabisa sub-district, KwaZulu-Natal.

Methods. Three hundred TB patients were randomly selected in a two-stage-sampling scheme with five primary healthcare clinic (PHC) sampling units selected with probability proportional to size. Data were collected using a structured questionnaire. We describe key availability and utilisation factors and analyse factors associated with being offered an HIV test in multiple regressions controlling for sex, age, education, employment and marital status.

Results. Most patients (75.2\%) received care for a first episode of TB, mainly pulmonary. Nearly all (94.3\%) were offered an HIV test during their current TB treatment episode, patients using their closest clinic being substantially more likely to have been offered HIV testing than those not using their closest clinic (adjusted odds ratio 12.79, $p=0.05$ ). About one-fifth (20.3\%) of patients did not take medication under observation, and 3.4\% reported missing taking their tablets at some stage. Average travelling time to the clinic and back was 2 hours, most patients (56.8\%) using minibus taxis.

Conclusion. We demonstrate high HIV testing rates among TB patients in a rural public programme, suggesting appropriate management of HIV-TB co-infected patients. We describe healthcare availability and utilisation factors that can inform the proposed district management teams for PHC re-engineering on areas needing improvement.
\end{abstract}

S Afr Med J 2013;103(4):237-240. DOI:10.7196/SAMJ.6423

The human immunodeficiency virus (HIV) continues to be a leading cause of death in sub-Saharan Africa, with South Africa (SA) having the highest number of people living with HIV. Countries with a high prevalence of HIV, mainly those in sub-Saharan Africa, have witnessed an increase in tuberculosis (TB) cases. High TB and HIV co-infection rates have been reported in SA. In 2009, more than $60 \%$ of TB patients in SA were reported to be co-infected with $\mathrm{HIV}^{[1]}$ the figure reaching nearly $80 \%$ in Hlabisa sub-district. ${ }^{[2]}$ HIV in TB patients increases mortality, promotes progression of latent and recently acquired TB to active $\mathrm{TB}$, and increases $\mathrm{TB}$ recurrence rates. ${ }^{[1]}$

TB patients face several challenges in accessing care, and an integrated service that includes HIV testing is most likely to ensure timely initiation of HIV treatment and optimal TB care. The need to integrate TB and HIV programmes was highlighted in 2004 by the World Health Organization (WHO)'s HIV and Stop TB departments, and from
2009 onwards WHO guidelines recommended that all HIV-positive patients with active TB start antiretroviral therapy (ART) immediately. ${ }^{[3]}$ Integrated TB/HIV activities, including HIV testing of all TB patients, can help ensure that HIV-positive TB patients are identified and treated appropriately and help prevent TB in HIV-positive patients, ${ }^{[1]}$ thereby accelerating universal access to comprehensive TB and HIV prevention, treatment and care.$^{[4]} \mathrm{HIV}$ testing rates among TB patients differ according to approaches used (voluntary or provider-initiated testing) and the setting, with higher rates being reported in integrated as opposed to vertical programmes. ${ }^{[5,6]}$

Vertical models are those in which HIV or TB services stand alone and cross-refer patients to the other service for testing and treatment. Programmes can be integrated in respect of funding, management, service delivery, and monitoring and evaluation of health systems ${ }^{[7,8]}$ From the patient's perspective it is most important 
that TB and HIV services are provided in the same facility. ${ }^{[6,9,10]}$ Facility-level integration in the delivery of TB and HIV services has been adopted in the primary healthcare clinics (PHCs) in the Hlabisa sub-district of KwaZulu-Natal. ${ }^{[6]}$ Benefits of such integration include increased satisfaction and reduced time and financial costs for patients receiving care for both diseases.

Understanding factors that influence availability and utilisation of healthcare is essential in providing comprehensive healthcare services. Multiple factors potentially affect healthcare utilisation among TB patients, including HIV testing uptake; some of these are patient socio-demographic characteristics, availability of services (e.g. directly observed treatment, short course (DOTS)), physical/ geographical variables (e.g. travel time, mode of transport), and organisational issues (e.g. opening times of clinics). The aim of this study was to investigate factors affecting healthcare availability and utilisation, including uptake of HIV testing, in TB patients in a programme devolved to rural PHCs in the Hlabisa sub-district.

\section{Methodology Setting}

The Department of Health TB (DoH-TB) control programme operates at the Hlabisa district hospital (DH) and 17 PHCs. The programme is integrated with the Hlabisa HIV Treatment and Care Programme (ART programme), which receives additional staff support and database management from the Africa Centre for Health and Population Studies (www.africacentre.com) ${ }^{[6,11]}$ The ART programme utilises a decentralised approach with integration of TB and HIV services to primary healthcare, linking treatment, care and prevention services. ${ }^{[11]}$

TB nurses at each PHC identify TB suspects and collect sputum, which is sent to the National Health Laboratory Service (NHLS) for acid-fast bacilli smear testing. All smear-positive patients are initiated onto the first-line standard TB regimen, and patients with negative smears (most of whom are HIV-infected) who remain symptomatic are referred to the $\mathrm{DH}$ for further clinical assessment and X-rays. ${ }^{[2,6]}$ The DoH-TB control programme adheres to national guidelines on the diagnosis, treatment and care of TB patients. All TB patients requiring inpatient services or those with complications, as well as those with multidrug-resistant (MDR) and extensively drugresistant (XDR) TB, are referred to the DH. After completing their hospitalisation (typically 1 month), patients are treated daily on an outpatient basis, with monthly clinic follow-up.

Since the end of 2009, a routine opt-out provider-initiated approach for HIV testing and counselling (PITC) has been in place at all PHCs in the sub-district, in addition to established voluntary counselling and testing. ${ }^{[11,12]} \mathrm{A}$ rapid HIV test is performed in patients who agree to be tested, and blood samples from HIV-positive patients are sent to the NHLS for CD4 cell counts. Results are available within 2 days and patients are requested to return to the clinic for their CD4 count results within a week of sample collection. Individuals who are not yet eligible for ART are monitored and followed up regularly. Prior to ART initiation, patients are screened for TB and other opportunistic infections, which are treated appropriately when diagnosed. ART initiation, treatment and care are offered according to the national guidelines.

As part of a larger multisite cross-sectional study called Researching Equity in ACcess to Healthcare (REACH), which was conducted in four sub-districts in SA in 2009 to examine barriers to healthcare access, we collected data on factors affecting healthcare availability and utilisation, including uptake of HIV testing in adults with TB attending PHCs in Hlabisa sub-district.

\section{Sampling and sample size}

We randomly selected patients in a two-stage-sampling scheme, with five PHC sampling units selected with probability proportional to size, and collected data through exit interviews with 300 patients utilising TB treatment at the selected PHCs. Three clinics that had some ongoing research activities were excluded from the sampling frame of the then 16 PHCs in the sub-district, to avoid over-burdening staff and patients.

\section{Data collection}

A structured questionnaire was administered to patients in isiZulu by four trained fieldworkers. Written consent was obtained from patients aged 18 years and above who volunteered to participate and had been on TB treatment for at least 2 months (by which time they would be expected to have completed the intensive phase of treatment). Those patients with MDR/XDR TB and who received treatment in hospital for the first 2 months were therefore also captured for this study, having been referred for follow-up treatment at the PHC. The study received ethical clearance from the University of KwaZulu-Natal (BE174/08).

\section{Statistical methods}

Summary statistics on the variables collected are set out in Table 1. Further, we regressed the binary variable indicating whether an HIV test was offered on a range of independent variables in multivariable logistic regression to gain insights on the predictors of HIV testing in this patient population. All analyses were done using STATA (Release Version 11, Stata Corporation, College Station, TX) and values of $p<0.05$ were considered significant.

\section{Results}

Table 1 shows the socio-demographic profile of patients utilising TB healthcare at the PHCs, as well as summary statistics on aspects of TB-related care. The majority of patients (75.2\%) were receiving care for a first episode of TB, in most cases pulmonary. Almost all patients (94.3\%) reported having been offered an HIV test during the current TB treatment episode, most had been diagnosed with TB at the same clinic at which they were receiving their TB treatment, and almost all (95.8\%) stated that they were able to access other healthcare services at the same facilities they utilised for their TB treatment. Although most patients were supported by DOTS, 20.3\% did not take their TB medication under observation.

Almost all patients (97.0\%) were accessing the clinic closest to their home for TB treatment. Self-reported reasons for not using the closest clinic were that the clinic they attended was closer to their workplace $(n=2)$, that they were offered good service at the present clinic $(n=1)$, that the nearest clinic did not give correct treatment $(n=1)$ or had too many patients and long queues $(n=1)$, lack of knowledge on how to change clinics $(n=1)$, treatment course almost completed and therefore no need to change clinics $(n=1)$, and changes in place of residence after initiating TB treatment at the present clinic $(n=2)$. More than a tenth of patients receiving TB care asserted that people in the community judged them negatively for attending for TB treatment.

Among the 9 patients who were not using their closest clinic, 2 $(22.2 \%)$ were female, the median age was 39 years (interquartile range (IQR) 35 - 48 years), 5 (55.6\%) had little or no education, 6 had never married (66.7\%) and 7 (77.8\%) were unemployed. Controlling for age, sex, education, marital status, employment and other factors associated with being offered an HIV test, patients using their closest clinic were substantially more likely to have been offered HIV testing than those not using their closest clinic (adjusted odds ratio 12.79, $p=0.05$ ). 
Table 1. Availability and utilisation of TB treatment and care services in primary healthcare clinics $(N=296)$

\section{Patient socio-demographic characteristics}

Gender, $n$ (\%)

Female

Age (years), $n$ (\%)

Mean $(95 \% \mathrm{CI})$

$<20-29$

$30-39$

$40-49$

$\geq 50$

Education, $n(\%)$

None

Primary

Secondary

Matric and higher

$53(17.9)$

Employed ( $n=295), n$ (\%)

Yes

Marital status, $n(\%)$

Never married

TB healthcare-related factors

Is this the first time you have had TB? ( $N=294), n(\%)$ Yes

Have you been offered an HIV test (during this current treatment episode), $n$ (\%)

Yes

Besides TB care, are you able to get the other health services you need in this facility? $(N=286), n(\%)$

Yes

Who checks that you have taken your TB treatment each day? $n(\%)$

The TB DOTS sister or counsellor

A community worker (community DOTS)

Someone at my place of work (workplace DOTS)

No one

Family members/partners/neighbours

Do you feel that people in the community judge you negatively for attending this facility for your $\mathrm{TB}$ treatment? $(N=257), n(\%)$

Yes

Since you first started coming to this facility, have you ever left without being helped? ( $N=296), n$ (\%)

Yes

During this current treatment episode, have you received treatment from a clinic other than this one? (N=293), $n(\%)$

Yes

Apart from the past 3 days, have you ever missed taking any tablets? $(N=293), n(\%)$

Yes
Table 1 (continued). Availability and utilisation of TB treatment and care services in primary healthcare clinics $(N=296)$

\begin{tabular}{l}
\hline TB healthcare-related factors \\
Are the opening hours of this clinic convenient \\
for you? $n(\%)$ \\
$\quad$ Yes \\
Is this the closest clinic to your home that offers \\
TB treatment? $n(\%)$ \\
$\quad$ Yes
\end{tabular}

$\mathrm{CI}=$ confidence interval.

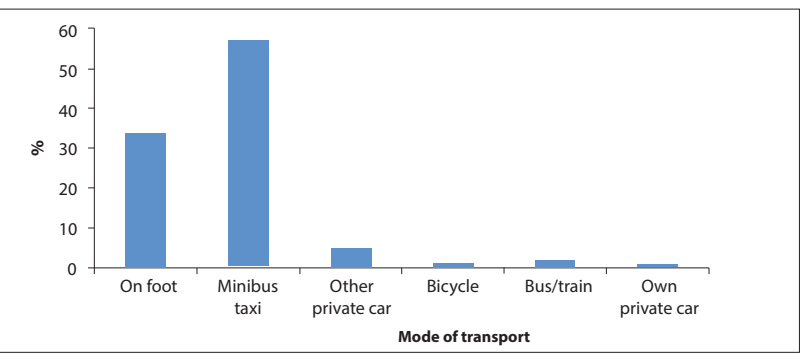

Fig. 1. Modes of transport used by patients (\%) to travel to the primary healthcare clinics to receive $T B$ treatment $(N=296)$.

The average travel time for patients attending a clinic was 2 hours for a return trip, and most of them used a minibus taxi (Fig. 1). The median time taken to travel (return trip) to the clinic on foot or by public transport was 90 minutes (IQR 60 - 180 minutes), by own car 60 minutes (IQR 40 - 75 minutes) and by other private car (a hired car or catching a lift) 120 minutes (IQR 60 - 120 minutes). ('Other private car' refers to a personal car that usually transports people from typically inaccessible areas where there is no public transport. Individuals hire these cars, or wait for them at pick-up points, or obtain lifts on their way to the destination point.)

We analysed the 17 patients who were not offered HIV testing according to socio-demographic characteristics, and found that 10 (58.8\%) were female and the median age was 33 years (IQR 30 49 years). A high proportion had never been married (13, 76.5\%) and were unemployed $(16,94.1 \%)$, and $3(17.7 \%)$ had no formal education. There was no statistically significant difference in the socio-demographic profile of those offered and not offered HIV testing, but statistical power to detect such differences was low due to the study sample size.

\section{Discussion and conclusion}

In an area with a high burden of TB and HIV, we found near-universal HIV testing among TB patients attending PHCs in a rural programme devolved to primary care level. Few people refused the offer of HIV testing once they had received counselling on the importance of knowing their HIV status for optimal general medical care and TB treatment. The HIV testing rate had risen to $94 \%{ }^{[13]}$ from the previously reported $88 \%,{ }^{[6,11]}$ with an age-sex profile comparable to previous studies in the area. ${ }^{[2,6]}$ There was no significant difference in terms of socio-demographic characteristics between patients not offered an HIV test and those offered one, indicating that health workers were following the TB guidelines on HIV screening satisfactorily and have adopted a successful PITC approach. 
Integration of TB and HIV services with primary healthcare services ensures patient accessibility to comprehensive healthcare and also strengthens health systems, in turn achieving improved universal access to health. ${ }^{[4,6,14]}$ Almost all patients in our study confirmed their ability to utilise the other healthcare services they required from the same facility at which they obtained their TB treatment. Joint delivery of TB and HIV in PHCs serves to support good accessibility (encompassing availability and utilisation of services) to comprehensive healthcare. However, some TB healthcare services need to be improved in respect of DOTS delivery to ensure sustained adherence to treatment. ${ }^{[4,14]}$ While the TB programme has not received the same level of support as the ART programme, ${ }^{[6]}$ further research is needed to understand the imperfections in TB DOTS coverage identified in this study. Significantly, the gap in the DOTS delivery system creates an opportunity to strengthen the current TB programme, e.g. through community-based linkages and DOTS support on the part of policymakers and district management teams, as proposed in efforts to reform the SA health system.

Patients generally use the clinic closest to their homes for TB treatment and HIV testing, implying that fear of stigma appears not to play a major role in their decision where to seek health care. This conclusion is supported by the fact that reasons for not using the clinic closest to home included better quality of care, shorter waiting time and queues, and closeness to place of work. The few patients who did not test for HIV at their closest clinic may have been diagnosed with resistant TB and hospitalised; they would have been offered HIV testing while treatment was initiated, and their results sent to the nearest clinic from the hospital. Our study area has experienced sustained high levels of both HIV and TB, as well achieving high coverage of treatment of both diseases; in 2010, 25\% of people in the area shared a household with someone receiving ART. ${ }^{[15]}$ Social exposure to people receiving treatment may have normalised attitudes and behaviours towards HIV and TB. Integration of HIV and TB services in PHCs, as well as PITC, is also likely to have reduced stigma.

The average travelling time for TB patients attending PHCs in Hlabisa sub-district was 2 hours for a return trip, and most used minibus taxis. The time losses arising from TB treatment are a potential threat to treatment uptake and retention. In this context, the time saved in utilising combined TB and HIV treatment at the same PHC, instead of in geographically separate locations, is likely to have had a positive impact on healthcare access by co-infected patients. Policymakers could consider providing subsidised transport for patients, and increasing the geographical density of PHCs in rural areas where possible.

In conclusion, this study demonstrates that in a devolved integrated public sector programme, TB and HIV services can be integrated successfully at the level of the rural PHC. The high HIV testing rates among TB patients indicate that patients co-infected with the two diseases are clearing the first hurdle to adequate treatment of HIV infection, and that service integration has the potential subsequently to ensure their appropriate management. However, research is needed on whether TB patients who test positive for HIV do indeed return for their CD4 count results and ART. Findings on factors affecting healthcare utilisation, such as DOTS, adherence and patient accessibility to clinics, could further inform activities deserving the focus of the district management teams proposed as part of the SA health system reform.

Author contributions. NC, MLN and TB contributed to conception and design, drafting and critical revision of the article. NC undertook acquisition, analysis and interpretation of data. All authors read and approved the final manuscript, and declare that they have no competing interests.
Acknowledgements. We would like to acknowledge the fieldworkers from the Africa Centre for Health and Population Studies who collected data used in this study (Mlungisi Mthetwa, Sibongiseni Mthetwa, Nomusa Mkhabela and Cynthia Ncube), the staff working at the clinics and the patients attending the PHCs for their support and participation in the study. We further thank the REACH principal investigators, team members and collaborating sites (Helen Schneider, Di McIntyre, Stephen Birch, John Eyles, especially for the comments and edits, Duane Blaauw, Bronwyn Harris, Pascalia Munyewende, Loveday Penn-Kekana, Mosa Moshabela, Susan Cleary, Vanessa Daries, Sheetal Silal, Veloshnee Govender, Jana Fried) for making this study possible. We are grateful to the REACH Cape Town team for the data entry and making data available to all sites.

Source of funding. This work was carried out with support from the Global Health Research Initiative (GHRI), a collaborative research funding partnership of the Canadian Institutes of Health Research, the Canadian International Development Agency, Health Canada, the International Development Research Centre and the Public Health Agency of Canada. The Africa Centre for Health and Population Studies, University of KwaZuluNatal, is supported by a grant from Wellcome Trust (082384/Z/07/Z). The Hlabisa HIV Treatment and Care Programme is funded by the generous support of the American people through the United States Agency for International Development (USAID) and the President's Emergency Plan (PEPFAR) under the terms of Award No. 674-A-00-08-0001-00. None of the funding organisations had any role in the design and conduct of the study, in the collection, analysis, and interpretation of the data, or in the preparation, review or approval of the manuscript.

\section{References}

1. World Health Organization. Global Tuberculosis Control: WHO report 2010. http://www.who.int/tb/ publications/global_report/en/ (accessed 30 May 2012).

2. Houlihan CF, Mutevedzi PC, Lessells RJ, Cooke GS, Tanser FC, Newell ML. The tuberculosis challenge in a rural South African HIV programme. BMC Infect Dis 2010;10:23. [http://dx.doi.org/10.1186/1471 2334-10-23]

3. World Health Organization. WHO Policy on TB Infection Control in Health-care Facilities, Congregate Settings and Households. Geneva: WHO, 2009. http://whqlibdoc.who.int/ publications/2009/9789241598323_eng.pdf (accessed 30 May 2012).

4. Gandhi NR, Moll AP, Lalloo U, et al. Successful integration of tuberculosis and HIV treatment in rural South Africa: the Sizongoba study. J Acquir Immune Defic Syndr 2009;50(1):37-43. [http://dx.doi org/:10.1097/QAI.0b013e31818ce6c4]

5. Sendagire I, Schreuder I, Mubiru M, van der Loeff MS, Cobelens F, Konde-Lule J. Low HIV testing rates among tuberculosis patients in Kampala, Uganda. BMC Public Health 2010;10(1):177. [http://dx.doi. among tuberculosis patients in

6. Wallrauch C, Heller T, Lessells R, Kekana M, Bärnighausen T, Newell ML. High uptake of HIV testing for

Wallrauch C, Heller T, Lessells R, Kekana M, Bärnighausen T, Newell ML. High uptake of HIV testing for
tuberculosis patients in an integrated primary health care HIV/TB programme in rural KwaZulu-Natal. S tuberculosis patients in an integ

Wallrauch C, Bärnighausen T, Heller T, Houlihan C, Newell ML. The white and the three-letter plague: integration of TB and HIV care in sub-Saharan Africa. Future HIV Therapy 2008;2(5):437-451. [http:// dx.doi.org/10.2217/17469600.2.5.437]

8. Atun R, Bennett S, Duran A. When Do Vertical (Stand-alone) Programmes Have a Place in Health Systems? Geneva: World Health Organization, 2008. http://www.who.int/management/district/services/ WhenDoVerticalProgrammesPlaceHealthSystems.pdf (accessed 12 October 2012).

9. South A, Legido-Quigley H, Khan P, Montgomery C, Fakoya A, Grant A. Evidence for action on HIV treatment and care systems integrating TB and HIV services. Policy briefing paper issue 6. 2010. http:// www.dfid.gov.uk/R4D/PDF/Outputs/HIV_AIDS/BriefingNo.6.pdf (accessed 30 May 2012).

10. Freidland $\mathrm{G}$, Harries $\mathrm{A}$, Coetzee D. Implementation issues in tuberculosis/HIV program collaboration and integration. 3 case studies J Infect Dis 2007:196(suppl 1):S114-S123. [http//dx doi.org/10.1086/518664] integration: 3 case studies. J Infect Dis 2007;196(suppl 1):S114-S123. [http://dx.doi.org/10.1086/518664] Houlihan CF, Bland RM, Mutevedzi PC, et al. Cohort profile: Hlabisa HIV treatment and care programme. oi.org/10.1093/ije/dyp402]

12. Houlihan CF, Maheswaran H, Thulare H, Heller T, Newell ML. Pilot of provider initiated testing and counselling in a rural primary health care clinic. Presented at the 4 th Southern African AIDS Conference, Durban, 31 March - 4 April 2009.

13. Chimbindi N, Bärnighausen T, Newell M-L. Almost universal coverage: HIV testing among TB patients in a rural public programme. Int J Tuberc Lung Dis 2012;16(5):708(i). [http://dx.doi.org/10.5588/ ijtld.11.0754]

14. Coetzee D, Hilderbrand K, Goemaere E, Matthys F, Boelaert M. Integrating tuberculosis and HIV care in the primary care setting in South Africa. Trop Med Int Health 2004;9(6):A11-15. [http://dx.doi. org/10.1111/j.1365-3156.2004.01259.x]

15. Bor J, Bärnighausen T, Newell C, Tanser F, Newell M-L. Social exposure to an antiretroviral treatment programme in rural KwaZulu-Natal. Trop Med Int Health 2011;16(8):988-994. [http://dx.doi.org/10.1111/ j.1365-3156.2011.02795.x]

Accepted 19 October 2012. 